\title{
Combining data fusion with multiresolution analysis for improving the classification accuracy of uterine EMG signals
}

\author{
Bassam Moslem ${ }^{1,2^{*}}$, Mohamad Diab ${ }^{3}$, Mohamad Khalil $^{2}$ and Catherine Marque
}

\begin{abstract}
Multisensor data fusion is a powerful solution for solving difficult pattern recognition problems such as the classification of bioelectrical signals. It is the process of combining information from different sensors to provide a more stable and more robust classification decisions. We combine here data fusion with multiresolution analysis based on the wavelet packet transform (WPT) in order to classify real uterine electromyogram (EMG) signals recorded by 16 electrodes. Herein, the data fusion is done at the decision level by using a weighted majority voting (WMV) rule. On the other hand, the WPT is used to achieve significant enhancement in the classification performance of each channel by improving the discrimination power of the selected feature. We show that the proposed approach tested on our recorded data can improve the recognition accuracy in labor prediction and has a competitive and promising performance.
\end{abstract}

Keywords: Multichannel analysis, Data fusion, Wavelet packet Transform (WPT), Uterine electromyogram (EMG), Labor detection

\section{Background}

Bioelectrical signals express the electrical functionality of different organs in the human body. The uterine electromyogram (EMG) signal, also called electrohysterogram (EHG), is one important signal among all bioelectrical signals. Recorded noninvasively from the abdominal wall of pregnant women, uterine EMG represents an objective and noninvasive way to quantify the uterine electrical activity. Studies have shown that uterine EMG can provide valuable information about the function aspects of the uterine contractility $[1,2]$. In addition, it is potentially the best predictor of preterm labor and of great value for the diagnosis of preterm delivery [3]. Although analyzing the uterine electrical activity represents an active research area, little attention has been brought to the classification of uterine EMG. In the literature, there exist only a few studies dealing with the classification of uterine EMG signals. In particular, Maner and Garfield

\footnotetext{
* Correspondence: bassam.moslem@utc.fr

${ }^{1}$ UMR CNRS 6600, laboratoire Biomécanique et Bio-ingénierie, Université de

Technologie de Compiègne, Compiègne 60205, France

${ }^{2}$ Azm Center for Research in Biotechnology and its applications, LASTRE

Laboratory, Lebanese University, Tripoli, Lebanon

Full list of author information is available at the end of the article
}

[4] used a kohonen method in order to classify uterine EMG data into term/preterm and labor/non-labor classes. Uterine contractions were quantified by finding the mean and the standard deviation of the power spectrum peak frequency, burst duration, number of bursts per unit time, and total burst activity. The approach applied on a total of 134 term and 51 preterm women yielded a classification accuracy of $80 \%$. Moreover, Lu et al. [5] presented a classification method based on the wavelet packet decomposition and a multilayer Perceptron (MLP) to differ between term and preterm data. Their study included 11 preterm and 28 term signals. They reported a classification accuracy of $64.1 \%$. Finally, in [3], Marque et al. used a multilayer perceptron and frequency-related parameters in order to differentiate between high risk contractions recorded at the same pregnancy terms on women who were proved to deliver preterm and normal contractions recorded on women who were proved to deliver at term. The results showed first that it was possible to detect a risk of preterm labor as early as 27 weeks of gestation with a classification accuracy of $87 \%$. 
However, all the previous studies were limited to the use of only two to four electrodes. A large number of experimental studies have shown that the uterus, as other biological systems, is complex both in its structure and functions [6,7]. This complexity arises from stochastic, nonlinear biological mechanisms interacting with a fluctuating environment. Therefore, reducing the recordings to one to two sensors of uterine EMG information may be very limiting. Recent studies on the electrophysiological activity of the uterine contractions have shown that multisensor recordings is a very promising technique that can offer better insight into the progression of pregnancy and can provide above all a fundamental contribution for predicting labor [8]. New parameters were derived from the multichannel recordings. In particular, synchronization [9-12] and propagation velocity [13-16] of the electrical activity of the uterine muscle were proven to be efficient tools to diagnose labor. Moreover, in terms of classification of uterine EMG signals, it was proven that the use of a data fusion-based approach can outperform the classification based on the use of a single electrodes $[17,18]$.

The aim of this study is to combine data fusion with multiresolution analysis based on the wavelet packet transform (WPT) in order to improve the recognition accuracy of multichannel uterine EMG data and attain the highest possible classification accuracy.

Herein, multiresolution analysis based on the WPT is first applied in order to improve the discrimination power of the selected feature. The WPT is a powerful signal decomposition technique adapted for the analysis of nonstationary signals such as the uterine EMG. WPT decomposes a signal into a set of finite orthonormal subspaces or packets. However, for solving classification problems, only few packets should be selected according to their discrimination power. The Local discriminant bases (LDB) algorithm introduced by Saito and Coifman [19] to address the problem of choosing the optimum set of signal subspaces for classification applications is therefore used. As a result, individual signals recorded by each sensor are first decomposed using their corresponding LDB tree structures. Then, relevant features are extracted from the highly discriminatory subspaces and fed to a classifier in order to classify the signals into one of two the classes (pregnancy and labor). These binary classification problems are solved by Support Vector Machines (SVM) classifiers with a Gaussian Radial Basis Function (RBF) kernel. Finally, a decision fusion rule based on the weighted majority voting (WMV) of the individual channels is applied. Figure 1 illustrates the generalized block diagram of the proposed approach.

The rest of the article is organized as follows. The following section is dedicated to the description of the methodology used in this study such as the LDB algorithm, the foundations of the SVM classifier as well as our decision-level data fusion method. Then, in Section "Results" we present the results obtained by applying our approach on real uterine EMG signals. These results are discussed in Section "Discussion". Finally, the article is ended with a conclusion section.

\section{Materials and methods}

\section{Database description}

The real uterine EMG signals used in this study were recorded on 32 women: 22 women were recorded during pregnancy (33 - 41 week of gestation, WG), 7 during labor (37 - 42 WG) and 3 during both pregnancy and labor $(33-42 \mathrm{WG})$. The mean and standard deviations of the gestational ages for pregnancy and labor were $34.14 \pm 3.94$ and $39.6 \pm 1.75$ weeks, respectively. Recordings were made in the University Hospital of Amiens in France and at the Landspitali University hospital in Iceland by using protocols approved by each ethical committee (ID-RCB 2011-A00500-41 in France and VSN 02-0006-V2 in Iceland). Recordings were performed by using a grid of 16 electrodes, arranged in a $4 \times 4$ matrix positioned on the women's abdomen [20]. The third electrode column was always put on the uterine median axis (Figure 2.a). Reference electrodes were placed on each hip of the woman. Signals were sampled at 200 $\mathrm{Hz}$. The recording device has an anti-aliasing filter with a cut-off frequency of $100 \mathrm{~Hz}$. After manual segmentation of the bursts of uterine electrical activity corresponding to contractions with the help of the standard tocodynamometer contraction data acquisition, we obtained 137 pregnancy contraction signals and 76 labor signals. The simultaneous tocodynamometer paper trace was digitalized to ease the segmentation of the bursts. In this study, in order to increase the signal-tonoise ratio, we considered vertical bipolar signals instead of monopolar ones. Our signals form thus a rectangular 3x4 matrix (Figure 2.b).

\section{Preprocessing}

Uterine EMG is a nonstationary signal that contains not only the useful information but also some unuseful parts such as the artifacts (mother ECG, fetal movements...). Therefore, the recorded signals cannot be used directly. Before extracting the features, three steps are performed in this study: (1) unwanted signals are removed by filtering the burst signals between 0.1 and $3 \mathrm{~Hz}$ [1]; (2) all signals are normalized by dividing each signal by its standard deviation in order to ensure that all features will have equal significance when they are applied to the committee machines [21]; (3) all burst signals were downsampled in order to reduce the number of the studied packets. 


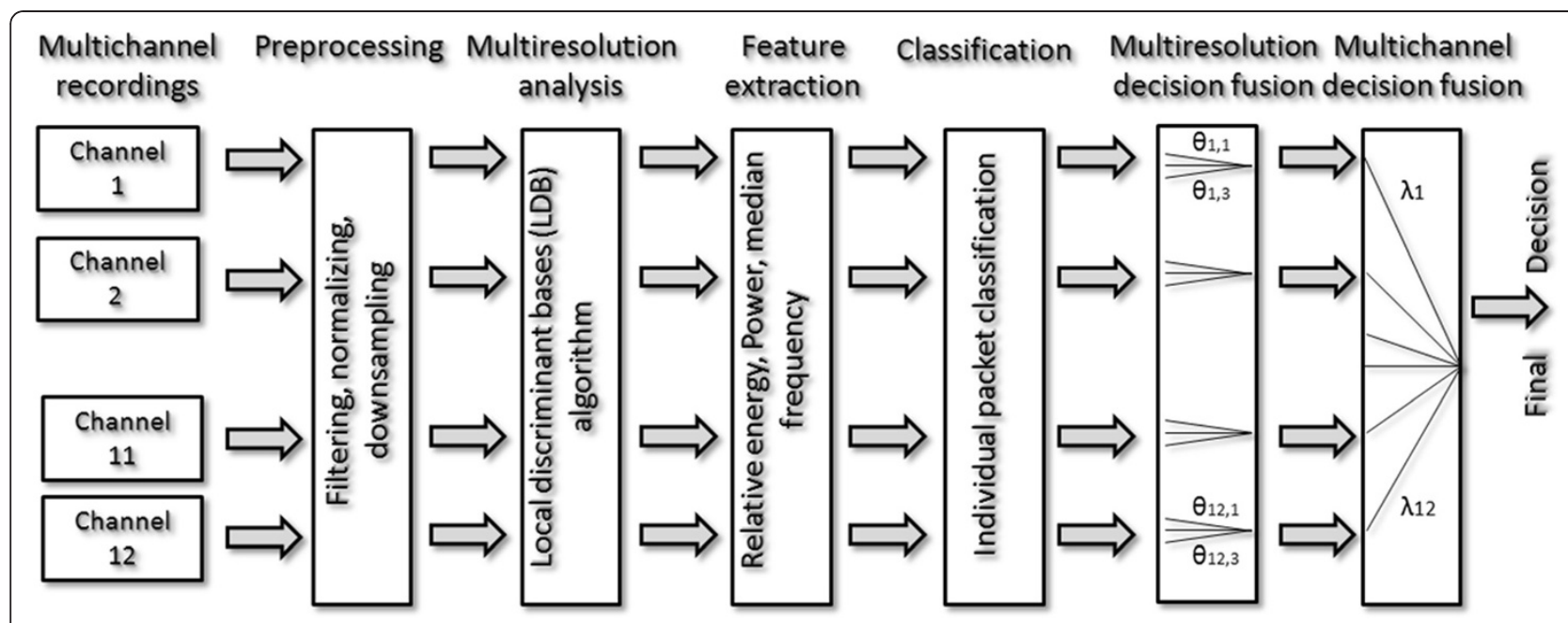

Figure 1 Block diagram of the proposed approach used for classifying multichannel uterine EMG signals.

The local discriminant bases (LDB) algorithm In this section, we describe the LDB algorithm that looks for a wavelet packet basis in a dictionary that best illuminates dissimilarities among classes by using some class separability or dissimilarity measure [19]. Let $\Omega_{0,0}$

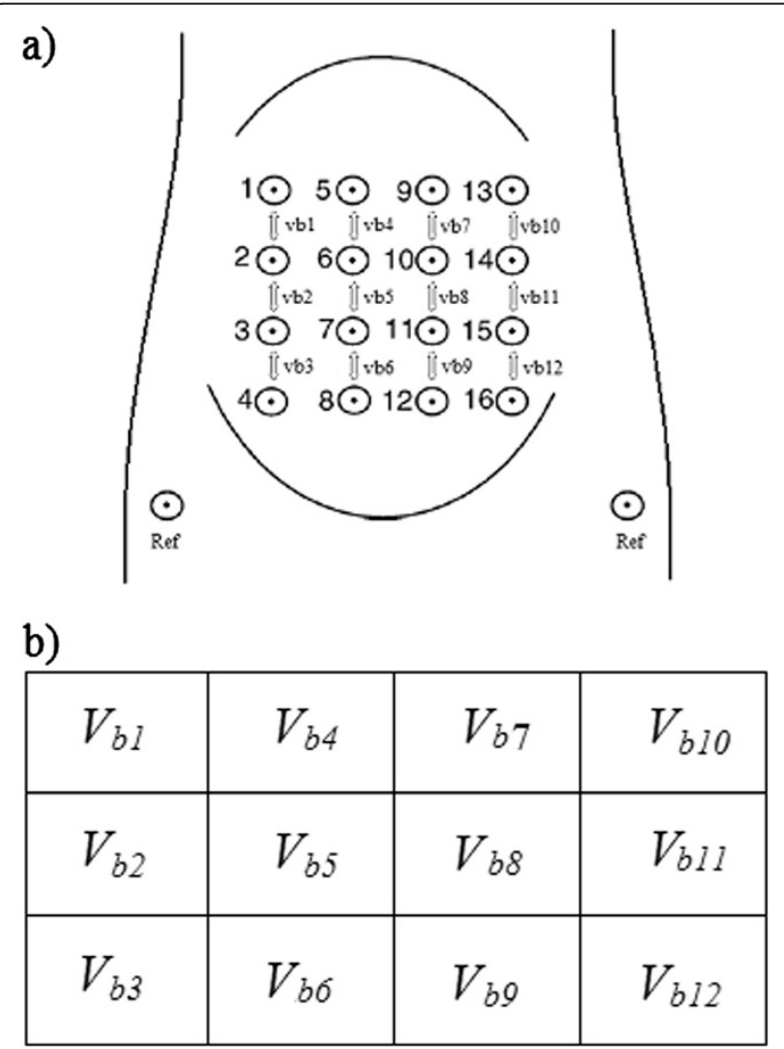

Figure 2 a. The $4 \times 4$ monopolar electrode configuration on the woman's abdominal wall. b. A $4 \times 3$ matrix representing all the 12 bipolar channels. denote the standard vector space in $\mathcal{R}^{n}$. Let $\Omega_{1,0}$ and $\Omega_{1,1}$ be the mutually orthogonal subspaces generated by the application of two projection operators $H$ and $G$ respectively to the parent space $\Omega_{0,0}$, i.e. $\Omega_{0,0}=\Omega_{1,0} \oplus \Omega_{1,1}$. These two operators correspond respectively to $h(n)$ and $g(n)$, the two impulse responses of low-pass and highpass analysis filters which correspond to the scaling function and the wavelet function respectively. The iterative decomposition process in the WPT generates subspaces of $\mathcal{R}^{n}$ of binary tree structure where the nodes of the tree represent subspaces with different frequency localization characteristics with $\Omega_{0,0}$ as the root node.

In general, at each level, the vector space is split by the operators $H$ and $G$ into two mutually orthogonal subspaces given by:

$$
\Omega_{j, k}=\Omega_{j+1,2 k} \oplus \Omega_{j+1,2 k+1}
$$

for $j=0,1 \ldots . . J, k=0 \ldots . .2^{j}-1$

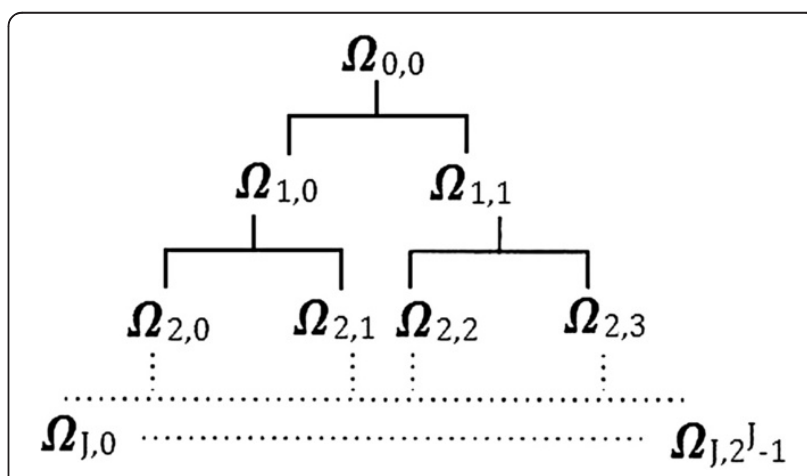

Figure $3 \mathrm{~A}$ binary tree associated with wavelet packet decompositions with $\Omega_{0,0}$ as the root node. 
This process repeats till the level $J$, giving rise to $2^{J}$ mutually orthogonal subspaces of equal bandwidth. Figure 3 shows the binary tree of the subspaces of $\Omega_{0,0}$.

Furthermore, each subspace $\Omega_{j, k}$ is spanned by $2^{n_{o}-j}$ basis vector $\left\{v_{j, k, l}\right\}_{l=0}^{2^{n_{0}-j}-1}$ where the triplet $(j, k, l)$ corresponds to scale, time localization and oscillation parameter, respectively, while $2^{n_{o}}$ corresponds to the length of the signal. Hence, the signal $x_{i}$ can be represented by a set of coefficients as:

$$
x_{i}=\sum_{j, k, l} \beta_{j, k, l} v_{j, k, l}
$$

where $i$ represents the time and $\beta_{j, k, l}$ represents the coefficients in each subspace represented by $(j, k)$.

\section{The dissimilarity measure}

The optimal choice of LDB for a given dataset is driven by the nature of the dataset and the dissimilarity measures used to distinguish between classes [22]. The choice is made in such a way that this dissimilarity measure should be able to bring out the differences between the signal classes in the time-frequency plane. It is interesting to note that a combination of multiple dissimilarity measures with varying complexity can also be used to achieve high classification accuracies.

In previous studies, it has been demonstrated that, throughout pregnancy, the energy of the recorded signals emerges significantly towards higher frequencies [23-25]. It was reported that there was a noticeable difference in the energy distribution of the uterine EMG signals between pregnancy and labor. Therefore, in this studies, our dissimilarity measure is defined as the difference in the normalized energy between the corresponding nodes of the different signal classes representing each class of contraction (pregnancy, labor).

$\mathcal{D}=\left|E_{j, k}^{1}-E_{j, k}^{2}\right|$ where $E_{j, k}^{1}$ and $E_{j, k}^{2}$ are the normalized energy of the corresponding nodes given by (3) for each class.

Based on the differences among these energy distributions, an orthonormal basis will allow the extraction of the distinguishing features among signal classes. Hence, the selection of an LDB from a given dictionary requires the calculation of the time-frequency (TF) energy maps for the whole binary tree.

The TF energy map of class $c$, denoted by $\Gamma_{c}$, is a table of real numbers specified by the triplet $(j, k, l)$ as

$$
\Gamma_{c}(j, k, l):=\frac{\sum_{i=0}^{2^{n_{o-1}}}\left(v_{j, k, l}^{T} x_{i}^{(c)}\right)}{\sum_{i=0}^{2^{n_{o-1}}}\left\|x_{i}^{(c)}\right\|^{2}}
$$

for $j=0 \ldots . J, k=0 \ldots . .2 J-l, l=0 \ldots . .2^{\left(n_{0}-j\right)}-1$ where $\sum_{i=0}^{2^{n_{o-1}}}\left\|x_{i}^{(c)}\right\|^{2}$ is the total signal energy belonging to class $c$.
The LDB algorithm results in only one complete best local discriminant basis for all the classes involved.

\section{Our LDB-based selection process}

To identify the relevant signal subspaces using the LDB algorithm, we follow the lines of Saito and Coifman in [19]. Given $N_{c}$ training signals consisting of the two classes of signals $\left\{\left\{x_{i}^{(c)}\right\}_{i=1}^{N_{c}}\right\}_{c=1}^{2}$ where each signal has a 12-channel resolution. Herein, the LDB selection process involves the use of the 12 channels of each training signal.

Step 1:

Construct a TF energy map $\Gamma_{c}$ for each of the two classes (pregnancy vs. labor) by using the training signals.

Step 2:

At each node, compute the discriminant measure among the two TF energy maps.

Step 3:

Prune the binary tree: eliminate children nodes if the sum of their discriminant measures is smaller than or equal to the discriminant measure of their parent node.

Step 4:

Order the most discriminant subspaces in increasing order of their power of discrimination.

\section{Calculation of the classifier's input parameters}

The selection of the input parameters or features is an important step for solving classification and pattern recognition problems [26]. The more information we have about the data, the more likely we would be able to succeed in assessing the true class for a single data item [27]. Furthermore, the task of feature selection is much more problem and domain dependent than is classification proper, and thus requires knowledge of the domain. Therefore, in our case, the selected features should also have a meaningful electrophysiological interpretation. This will help improve also our understanding of the electrophysiological dynamics underlying the changes of the uterine electrical activity during pregnancy and thus, improve our pregnancy monitoring scheme. Herein, we wish to favor a small number of features, which might lead to simpler decision regions and a classifier easier to train.

The energy and the frequency content of the uterine contractions are classical features which were included in many studies to characterize the uterine activity. Therefore, in this work, three features were extracted from the LDBs: power of the contraction, median frequency and the proportion of the energy contained in 
the selected packet (relative energy). These features were chosen based on previous studies [2,23,28] and showed that they may have either some predictive worth or some physiological significance.

\section{Support vector machines (SVM)}

SVM is a powerful classification technique based on the statistical learning theory [29]. It is primarily a two-class classifier. However, multiclass classification is accomplished by combining multiple binary SVMs.

The optimization criterion is the width of the margin between the classes, i.e., the empty area around the decision boundary (the separating hyperplane) defined by the distance to the nearest training patterns. These patterns, called support vectors, finally define the classification function.

Let $w$ and $b$ denote the weight vector and the bias in the optimal hyperplane, respectively, the corresponding hyperplane can be defined as:

$$
w^{T} \cdot y+b=0
$$

On a pattern $y$, the discriminant function of a binary SVM is given by:

$$
f(Y)=\operatorname{sgn}\left(\sum_{i=1}^{N} \alpha_{i} y_{i} K\left(y_{i}, y\right)+b\right)
$$

where $N$ is the number of learning patterns, $y_{i}$ are the training pattern with corresponding labels $y_{i} \in\{-1,+1\}(-1$ and +1 stand for the negative and positive classes, respectively), $b$ is a bias, and $K\left(y_{i}, y\right)$ is a kernel function mapping the input vectors into an expanded feature space.

The coefficients $\alpha_{i}$ are obtained by solving the following quadratic optimization problem:

$$
L(\alpha)=\sum_{i=1}^{N} \alpha_{i}-\left(\frac{1}{2}\right) \sum_{i=1}^{N} \sum_{j=1}^{N} \alpha_{i} \alpha_{j} y_{i} y_{j} K\left(y_{i}, y_{j}\right)
$$

subject to two constraints given in (6) and (7):

$$
\begin{aligned}
& , i=1, \ldots, N \\
& \sum_{i=1}^{N} \alpha_{i} y_{i}=0
\end{aligned}
$$

$K\left(y_{i}, y_{j}\right)$ can be written as $K\left(y_{i}, y\right)=\phi(y) . \phi\left(y_{i}\right)$ where $\phi(y)$ is the feature vector in the expanded feature space and may have infinite dimensionality. Linear kernel, sigmoid kernel, polynomial kernel, and RBF kernel are most commonly used kernel functions. In this study, we used Gaussian Radial Basis Function (RBF) kernel of the following form:

$$
K\left(y_{i}, y_{j}\right)=\exp \left(\sum_{i} \frac{\left(y_{i}-y_{j}\right)^{2}}{2 \sigma_{i}^{2}}\right)
$$

where $\sigma$ is the kernel width parameter.

\section{Decision-level data fusion}

Multisensor data fusion refers to the acquisition, processing and synergistic combination of information gathered by various sensors to provide a better understanding of a phenomenon and to yield more accurate results for information processing problems [30]. Data fusion has been successfully used in several applications in military and non-military domains such as classifying hyperspectral images [31], automatic emotion recognition [32], biomedical signal processing [33,34].

Data fusion can be done at one of three possible levels: direct fusion of sensor data at the first level (also called central fusion), representation of sensor data via feature vectors, with subsequent fusion of the feature vectors at the second, or processing each sensor to achieve highlevel inferences decisions, which are subsequently combined [35]. Noteworthy, each of these approaches utilizes different fusion techniques.

Combining multisource data from several sensors is believed to offer several advantages over data from a single sensor. First, combining the observations from several identical sensors offers an improved classification rate. A second advantage gained by using multiple sensors is improved observability of the studied physiological system [35]. Finally, using many decisions is generally more stable and often more useful, in terms of robustness to unknown pattern samples that do not appear in the training stage, then using a single decision. Herein, we use a decision-level data fusion method which combines sensor information after each sensor has made a preliminary decision. Examples of decisionlevel fusion methods include weighted decision methods (voting techniques), classical inference, Bayesian inference, and Dempster-Shafer's method [30,35]. In this study, the decision-level fusion method is based on the observation that the channel with a high accuracy should have more influence on the decision making than the channel with a lower accuracy. The same principle is also applied to the target packets. Weights, noted $\theta_{i, j}$ and $\lambda_{\mathrm{i}}(i=1, . .12 ; j=1, . .3)$, are therefore associated to the target packets and channels respectively to express quantitatively their classification reliability. Herein, reliability measures which rank the channels and packets according to their goodness are obtained heuristically based on the classification success of a trial dataset during an independent training phase [36]. The reason of 


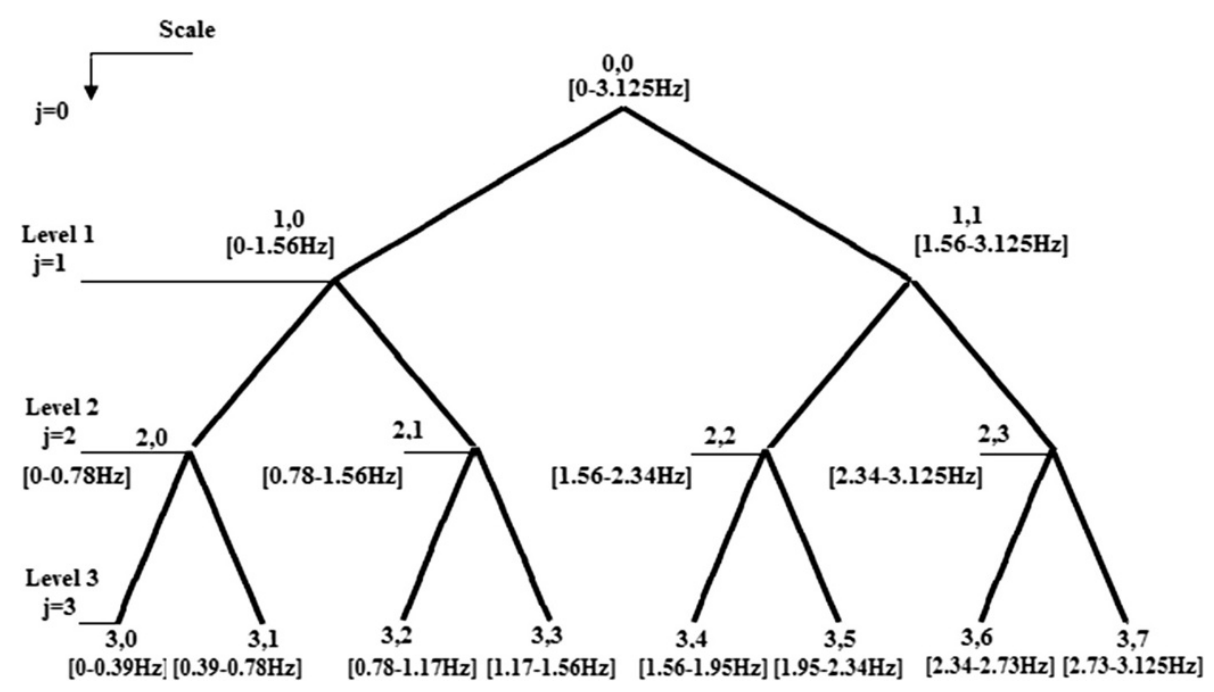

Figure 4 The wavelet packet decomposition tree with three levels and the respective frequency band for each packet.

calculating the values of the weights heuristically is due mainly to the fact that we lack complete knowledge of the properties of the different channels. Further, the final decision was based on the WMV decision of each component classifier combined: for our $m$-channel problem $\left\{d_{1}, d_{2}, \ldots, d_{m}\right\}$, (where $m=12$ ), where $d_{p}$ represents the decision of channel $p$, we define our decision fusion rule as follows:

$$
d_{f}=\sum_{p=1}^{m} d_{p} \lambda_{p}
$$

If $\left(d_{f}>0\right)$, then the decision is class 1 (pregnancy). However, if, $\left(d_{f} \leq 0\right)$ the decision is class 2 (labor).

However, in order to reduce the error due to the small sample size of training data, we have used the leave-oneout cross validation method where a single observation taken from the entire samples is used as the validation data while the remaining observations are used for training the classifier. This is repeated such that each observation in the samples is used once in the validation data [26]. In this phase, the training sets consisted each of an equal number of trials from the two classes (pregnancy, labor). Otherwise, the classifier will be biased toward the class from which it has seen most feature vectors [27]. Finally, we used an independent test set on which we evaluated our approach's classification success rate. The results of the classification are given in terms of correct classification accuracy which is the number of samples correctly classified divided by the total number of samples used.

\section{Results}

The total number of contractions included in this paper is 137 pregnancy and 76 labor contractions. Of these, a first 30 signals $\left(N_{c}=30\right)$ randomly chosen of each class was used as a first independent training dataset in order to find the most discriminant packets. Since the energy of the uterine EMG signals is located in a very limited bandwidth $(0.1$ and $3 \mathrm{~Hz})$ [1], all signals were decomposed only into a three-level ( $j=1$ to 3 ) wavelet packet tree. The decomposition led to eight packets at the third level of bandwidth $0.39 \mathrm{~Hz}$ each. Figure 4 shows the wavelet packet decomposition tree with the three levels. In this figure, the corresponding bandwidth is indicated at each node.

The Symlet 5 was chosen as the mother wavelet based on previous research results [37]. The three highest discriminant subspaces were chosen as the LDBs. After applying the LDB algorithm, packets $(3,0),(3,1)$ and $(2,1)$ were chosen as the most discriminant packets. These packets correspond to the frequency bands $[0-0.39 \mathrm{~Hz}]$, $[0.39-0.78 \mathrm{~Hz}]$, and $[0.78-1.56 \mathrm{~Hz}]$, respectively and con-

Table 1 Classification results of pregnancy and labor contractions of the second training dataset for each packet

\begin{tabular}{|c|c|c|c|c|}
\hline Packet & $\begin{array}{l}\text { Classification accuracy of } \\
\text { pregnancy contractions } \\
\text { (in \%) }\end{array}$ & $\begin{array}{l}\text { Classification accuracy of } \\
\text { labor contractions } \\
\text { (in \%) }\end{array}$ & $\begin{array}{c}\text { Overall Classification } \\
\text { accuracy } \\
\text { (in \%) }\end{array}$ & $\theta_{i, j}$ \\
\hline$(3,0)$ & 68.4 & 72.3 & 70.3 & 0.703 \\
\hline$(3,1)$ & 93.4 & 30 & 61 & 0.61 \\
\hline$(2,1)$ & 45.9 & 57.8 & 74.3 & 0.743 \\
\hline
\end{tabular}


Table 2 Classification results of pregnancy and labor contractions of the third training dataset for each channel

\begin{tabular}{|c|c|c|c|c|}
\hline Channel & $\begin{array}{l}\text { Classification accuracy of } \\
\text { pregnancy contractions } \\
\text { (in \%) }\end{array}$ & $\begin{array}{l}\text { Classification accuracy of } \\
\text { labor contractions } \\
\text { (in \%) }\end{array}$ & $\begin{array}{c}\text { Overall classification } \\
\text { accuracy } \\
\text { (in \%) }\end{array}$ & $\lambda_{i}$ \\
\hline $\mathrm{Vb}_{1}$ & 89.4 & 39.4 & 64.4 & 0.644 \\
\hline $\mathrm{Vb}_{2}$ & 64.4 & 50 & 57.2 & 0.572 \\
\hline $\mathrm{Vb}_{3}$ & 85.5 & 61.8 & 73.6 & 0.736 \\
\hline $\mathrm{Vb}_{4}$ & 71 & 44.7 & 57.9 & 0.579 \\
\hline $\mathrm{Vb}_{5}$ & 86.8 & 47.3 & 67.1 & 0.671 \\
\hline $\mathrm{Vb}_{6}$ & 80.2 & 40.7 & 60.5 & 0.605 \\
\hline $\mathrm{Vb}_{7}$ & 82.8 & 30.2 & 56.5 & 0.565 \\
\hline $\mathrm{Vb}_{8}$ & 84.2 & 38.1 & 61.1 & 0.611 \\
\hline $\mathrm{Vb}_{9}$ & 81.5 & 53.9 & 67.7 & 0.677 \\
\hline $\mathrm{Vb}_{10}$ & 94.7 & 48.6 & 71.7 & 0.717 \\
\hline $\mathrm{Vb}_{11}$ & 85.5 & 47.3 & 66.4 & 0.664 \\
\hline $\mathrm{Vb}_{12}$ & 81.5 & 60.5 & 71 & 0.71 \\
\hline
\end{tabular}

tainmore than $98 \%$ of the energy of the uterine EMG signals [23].

Next, a second and a third datasets consisting each of 20 signals randomly chosen from each of the two classes of contractions (pregnancy, labor) were used to determine the weights $\theta_{i, j}$ and $\lambda_{i}$ respectively. Features were extracted from the target subspaces and fed to an SVM classifier. Thereafter, the weights $\theta_{i, j}$ were assigned according to the classification results of each packet. The classification accuracies of the training data for each packet as well as the corresponding weights $\theta_{i, j}$ are indicated in Table 1.

Furthermore, the classification accuracies of the training set for each channel are shown in Table 2. The results show that the classification performance varies from one channel to another. Specifically, channel $V b_{3}$ had the highest predictive value (73.6\%) while for channel $\mathrm{Vb}_{7}$ had the worst (56.5\%). As a result, the highest weight was assigned to channel $V b_{3}$ while the lowest weight was assigned to channel $V b_{7}$. Based on the classification accuracy obtained on the trials in the training set, the weights $\lambda_{i}$ were therefore assigned to each channel. The values of the weights $\lambda_{i}$ are also indicated in Table 2 .

Finally, the trained network was used to classify the test signals. The network was tested by using 97

Table 3 Classification results of pregnancy and labor contractions for each channel for the test data by using a weighted decision fusion rule

\begin{tabular}{cc}
\hline Classification accuracy of pregnancy contractions & $\begin{array}{c}\text { Weighted decision } \\
\text { fusion method } \\
\text { (in \%) }\end{array}$ \\
\hline Classification accuracy of labor contractions & 95.2 \\
\hline Overall classification accuracy & 89.3 \\
\hline
\end{tabular}

pregnancy contractions and 36 labor contractions (including the first training dataset used to determine the most discriminant packets). The final decisions of the individual channels were fused by using the WMV fusion rule. The final classification results are presented in Table 3. By using a weighted decision fusion method, the overall classification accuracy was higher than any of the individual channels. An overall classification accuracy of 92.4\% was achieved. The accuracy was as high as $95.2 \%$ for pregnancy contractions and $89.3 \%$ for labor contractions as indicated in Table 3 . The high percentage of correctly classified labor/non-labor events indicates explicitly just how efficient this approach method is at detecting labor. Therefore, it can be seen that multichannel recordings can remarkably increase the classification rate of uterine EMG signals for both pregnancy and labor contractions.

\section{Discussion}

Numerous studies have analyzed the uterine EMG recordings associated with pregnancy and labor. It has been proved that it is of interest to offer a good insight into the process of pregnancy and labor and may also be used to predict the risk of preterm labor. Herein, we confirmed the importance of uterine EMG for detecting the onset of labor by classifying uterine EMG signals into 2 classes (pregnancy vs. labor). The results of this study showed that this technique may replace the methods currently available to clinicians to diagnose labor, such as internal and external tocography, cervical change evaluation by ultrasound examination [38].

Herein, we have used a matrix of 16 electrodes to improve the classification accuracy of recorded uterine electrical activity. The first point that emerges is that, although there was a correlation between the electrical activities recorded at different sites, the characteristics of 
the recorded signal depended on the position of the recording electrode. The results revealed first that the classification performance varied from one channel to another. One possible explanation of this observable fact is that the characteristics of the uterine electrical activity are influenced by the position of the recording electrode: on the one hand, the distance between the recording position on the skin and the signal source in the myometrium is reduced at the median axis with respect to other electrodes $[3,39,40]$. There are more visceral tissues between the skin and the uterus at the extremities than on the middle of the median axis of woman's abdomen [40]. On the other hand, in the region surrounding the median axis, the position of the uterus relative to the abdominal wall is constant even during contraction [3]. It was demonstrated thereafter that the main effect of the electrode position, in terms of tissues depth below the recording site and distance of electrodes to the potentials source, concerns the attenuation of the high frequencies which creates a strong attenuation of the signal energy [3] which explains the rise of the highfrequency content of the action potentials throughout pregnancy and at the onset of labor reported in many studies $[1,24]$. Therefore, the difference of the features values between the two classes is less significant at the median axis compared to other channels located at the extremities of the recording matrix. Therefore, electrodes positioned on the extremities are more sensible to these variations than the channels located at the center of the matrix and had more influence on the decision making than the ones positioned on the median axis. As a result, when a decision fusion rule was applied, an improved accuracy of the classification decision compared to a decision based on any of the individual data sources alone was obtained. Furthermore, based on the fact that there was variability between the classification accuracies of the different channels, a decision fusion rule based on the WMV may be more convenient for combining the decisions than other rules such as the majority voting as concluded in [41]. It is important also to note that, due to the complexity of the analyzed data, a SVM classifier with a RBF kernel which is known to be a strong classifier should be used as the component classifier of the network. When tested on the same data, SVM classifier with a RBF kernel yielded better classification results than a neural network of the same kernel function $[18,41]$.

On the other hand, the use of the multiresolution analysis and the LDB algorithm that selects a basis from a dictionary that illuminates the dissimilarities among the two classes presented an important preprocessing step for increasing the discriminatory powers of the extracted features. Despite the use of simple and classical parameters, this method yielded better results than any of the reported accuracies when compared with earlier results [3-5].

We conclude therefore that the combination of multiresolution analysis with data fusion can be a very powerful approach when nature of data to be classified is very complex as in the case of the uterine EMG signals.

Finally, although still to be tested, we believe that the results of our approach may be improved by using a more advanced decision-level fusion method such as the Bayesian inference and Dempster-Shafer's method. Also, other sophisticated uterine EMG parameters not considered in this study (e.g. propagation velocity, fractal dimension, complexity [42],...) may help improve these results. Finally, it is important to note that the use of the LDB algorithm, as well as the determination of the different weights requires a large number of training signals; therefore, by recording more signals, this technique may yield better classification results. As our ultimate goal is to improve the classification accuracy of uterine EMG signals in order to help detect preterm labor, we find these results to be very useful.

\section{Conclusion}

The classification of uterine EMG signals recorded by using multiple sensors was addressed. From this study, we can conclude first that the classification of uterine EMG signals can be improved by applying a decisionlevel data fusion rule. Also, we can conclude that multiresolution analysis based on the wavelet packet transform a data fusion seems to be an effective method for improving the classification of labor/non-labor signals. Although simple parameters and a small training dataset were used, the network demonstrated good performance on complex classification problems. As our goal is to improve the classification accuracy of uterine EMG data, we find the classification results very promising compared to previously reported results.

\section{Competing interests}

There are no competing interests for any of the authors to declare.

\section{Author details}

${ }^{1}$ UMR CNRS 6600, laboratoire Biomécanique et Bio-ingénierie, Université de Technologie de Compiègne, Compiègne 60205, France. ${ }^{2}$ Azm Center for Research in Biotechnology and its applications, LASTRE Laboratory, Lebanese University, Tripoli, Lebanon. ${ }^{3}$ College of Engineering, Bio-instrumentation Department, Rafik Hariri University (RHU), Meshref, Lebanon.

Received: 30 October 2011 Accepted: 7 July 2012

Published: 3 August 2012

\section{References}

1. D Devedeux, C Marque, S Mansour, G Germain, J Duchene, Uterine electromyography: a critical review. Am. J. Obstet. Gynecol. 169, 1636-1653 (1993)

2. B Moslem, M Hassan, M Khalil, C Marque, M Diab, Monitoring the Progress of Pregnancy and Detecting Labor Using Uterine Electromyography. International Symposium on Bioelectronics and Bioinformatics (RMIT University, School of Electrical and Computer Engineering, Melbourne, 2009), pp. 160-163 
3. CK Marque, J Terrien, S Rihana, G Germain, Preterm labour detection by use of a biophysical marker: the uterine electrical activity. BMC Pregnancy Childbirth 1(7 Suppl), S5 (2007)

4. WL Maner, RE Garfield, Identification of human term and preterm labor using artificial neural networks on uterine electromyography data. Ann. Biomed. Eng. 35, 465-473 (2007)

5. N Lu, J Wang, I McDermott, S Thornton, M Vatish, H Randeva, Uterine electromyography signal feature extraction and classification. Int J Modelling, Identification and Control. 6, 136-146 (2008)

6. H Maul, WL Maner, GR Saade, RE Garfield, The physiology of uterine contractions. Clin. Perinatol. 30, 665-676 (2003). v

7. S Wray, S Kupittayanant, A Shmygol, RD Smith, T Burdyga, The physiological basis of uterine contractility: a short review. Exp. Physiol. 86, 239-246 (2001)

8. M Hassan, J Terrien, A Alexandersson, B Karlsson, C Marque, Improving the classification rate of labor vs. normal pregnancy contractions by using EHG multichannel recordings. 32nd Annual International Conference of the IEEE EMBS Buenos Aires (2010)

9. C Marque, J Duchene, Human abdominal EHG processing for uterine contraction monitoring. Biotechnology 11, 187-226 (1989)

10. M Hassan, J Terrien, B Karlsson, C Marque, Spatial analysis of uterine EMG signals: evidence of increased in synchronization with term. Conf Proc IEEE Eng Med Biol Soc, 6296-6299 (2009). 2009/12/08 ed

11. M Hassan, J Terrien, B Karlsson, C Marque, Interactions between Uterine EMG at Different Sites Investigated Using Wavelet Analysis: Comparison of Pregnancy and Labor Contractions. EURASIP Journal on Advances in Signal Processing. 2010, 9 (2010)

12. J Terrien, T Steingrimsdottir, C Marque, B Karlsson, Synchronization between EMG at Different Uterine Locations Investigated Using Time-Frequency Ridge Reconstruction: Comparison of Pregnancy and Labor Contractions. EURASIP Journal on Advances in Signal Processing, 1-10 (2010). 2010

13. C Rabotti, M Mischi, SG Oei, JW Bergmans, Noninvasive estimation of the electrohysterographic action-potential conduction velocity. IEEE Trans Biomed Eng 57, 2178-2187 (2010)

14. M Mischi, C Rabotti, $L$ Vosters, SG Oei, JM Bergmans, Electrohysterographic conduction velocity estimation. Conf Proc IEEE Eng Med Biol Soc. 2009, 6934-6937 (2009)

15. M Lucovnik, WL Maner, LR Chambliss, R Blumrick, J Balducci, Z NovakAntolic et al., Noninvasive uterine electromyography for prediction of preterm delivery. Am. J. Obstet. Gynecol. 204, 228 (2011). e1-10

16. C Rabotti, M Mischi, JO van Laar, GS Oei, JW Bergmans, Inter-electrode delay estimators for electrohysterographic propagation analysis. Physiol. Meas. 30, 745-761 (2009)

17. B Moslem, MO Diab, M Khalil, C Marque, Classification of multichannel uterine EMG signals by using unsupervised competitive learning. Signal Processing Systems (SiPS), 267-272 (2011). IEEE Workshop on2011

18. B Moslem, MO Diab, C Marque, M Khalil, Classification of multichannel uterine EMG signals (Engineering in Medicine and Biology Society,EMBC, 2011), pp. 2602-2605. Annual International Conference of the IEEE2011

19. N Saito, RR Coifman, Local discriminant bases and their applications. Journal of Mathematical Imaging and Vision. 5, 337-358 (1995)

20. B Karlsson, J Terrien, V Gudmundsson, T Steingrimsdottir, C Marque, Abdominal EHG on a 4 by 4 grid: mapping and presenting the propagation of uterine contractions, in 11th Mediterranean Conf on Med and Biomed Eng and Comp, ed. by T. Jarm, P. Kramar, A. Zupanic (Springer, Berlin Heidelberg, 2007), pp. 139-143

21. GJ Lehman, SM McGill, The importance of normalization in the interpretation of surface electromyography: a proof of principle. J. Manipulative Physiol. Ther. 22, 444-446 (1999)

22. K Umapathy, S Krishnan, Feature analysis of pathological speech signals using local discriminant bases technique. Medical and Biological Engineering and Computing 43, 457-464 (2005)

23. B Moslem, M Khalil, C Marque, MO Diab, Energy Distribution Analysis of Uterine Electromyography Signals. Journal of Medical and Biological Engineering. 30, 361-366 (2010)

24. C Marque, JM Duchene, S Leclercq, GS Panczer, J Chaumont, Uterine EHG processing for obstetrical monitoring. IEEE Trans Biomed Eng 33, 1182-1187 (1986)
25. D Schlembach, WL Maner, RE Garfield, H Maul, Monitoring the progress of pregnancy and labor using electromyography. Eur. J. Obstet. Gynecol. Reprod. Biol. 144, S33-S39 (2009)

26. R Duda, P Hart, D Stork, Pattern Classification, 2nd edn. (Wiley-Interscience, 2000)

27. AK Jain, RPW Duin, M Jianchang, Statistical pattern recognition: a review. Pattern Analysis and Machine Intelligence, IEEE Transactions on. 22, 4-37 (2000)

28. I Verdenik, M Pajntar, B Leskosek, Uterine electrical activity as predictor of preterm birth in women with preterm contractions. Eur. J. Obstet. Gynecol: Reprod. Biol. 95, 149-153 (2001)

29. VN Vapnik, An overview of statistical learning theory. IEEE Trans. Neural Netw. 10, 988-999 (1999)

30. PK Varshney, Multisensor data fusion. Electronics \& Communication Engineering Journal. 9, 245-253 (1997)

31. J Benediktsson, I Kanellopoulos, Classification of multisource and hyperspectral data based on decision fusion. IEEE Trans Geosci Remote Sens 37, 1367-1377 (1999)

32. J Kim, E André, in Fusion of Multichannel Biosignals Towards Automatic Emotion Recognition Multisensor Fusion and Integration for Intelligent Systems, ed. by H. Hahn, H. Ko, S. Lee (Springer, Berlin Heidelberg, 2009), pp. 55-68

33. BO Peters, G Pfurtscheller, H Flyvbjerg, Automatic differentiation of multichannel EEG signals. IEEE Trans Biomed Eng 48, 111-116 (2001)

34. L Gupta, B Chung, MD Srinath, DL Molfese, H Kook, Multichannel fusion models for the parametric classification of differential brain activity. IEEE Trans Biomed Eng 52, 1869-1881 (2005)

35. D Hall, Llinas J (CRC Press, Handbook of multisensor data fusion, 2001)

36. JA Benediktsson, PH Swain, Consensus theoretic classification methods. Systems, Man and Cybernetics, IEEE Transactions on. 22, 688-704 (1992)

37. M Khalil, J Duchene, Uterine EMG analysis: a dynamic approach for change detection and classification. IEEE Trans Biomed Eng 47, 748-756 (2000)

38. RE Garfield, K Chwalisz, L Shi, G Olson, GR Saade, Instrumentation for the diagnosis of term and preterm labour. J. Perinat. Med. 26, 413-436 (1998)

39. C Rabotti, M Mischi, JO van Laar, GS Oei, JW Bergmans, Estimation of internal uterine pressure by joint amplitude and frequency analysis of electrohysterographic signals. Physiol. Meas. 29, 829-841 (2008)

40. J Terrien, S Rihana, J Gondry, Marque C (Modeling the effects of the electrodes position on the surface EMG characteristics, IFAC Modeling and Control in Biomedical Systems, 2006)

41. B Moslem, M Khalil, MO Diab, A Chkeir, C Marque, A multisensor data fusion approach for improving the classification accuracy of uterine EMG signals. Electronics, Circuits and Systems (ICECS) (2011), pp. 93-96. 18th IEEE International Conference on 2011

42. B Moslem, M Khalil, C Marque, MO Diab, Complexity analysis of the uterine electromyography. Engineering in Medicine and Biology Society (EMBC) (2010), pp. 2802-2805. Annual International Conference of the IEEE 2010

doi:10.1186/1687-6180-2012-167

Cite this article as: Moslem et al:: Combining data fusion with multiresolution analysis for improving the classification accuracy of uterine EMG signals. EURASIP Journal on Advances in Signal Processing 2012 2012:167.

\section{Submit your manuscript to a SpringerOpen ${ }^{\circ}$ journal and benefit from:}

- Convenient online submission

- Rigorous peer review

- Immediate publication on acceptance

- Open access: articles freely available online

- High visibility within the field

- Retaining the copyright to your article

Submit your next manuscript at springeropen.com 